\title{
Vibrational Bands of the 2-Butyn-1-yl Radical
}

\author{
Glenna J. Brown, Martha J. Ellis, Thaddeus D. Martin, Laura R. McCunn* \\ Department of Chemistry, Marshall University \\ 1 John Marshall Dr. Huntington, West Virginia 25755 \\ *corresponding author: mccunn@,marshall.edu, 304-696-2319
}

Table S1. B3LYP/6-311+G(3df,2pd) optimized geometry of 2-butyn-1-yl

Table S2. B3LYP/6-311+G(3df,2pd) optimized geometry of 2-butyne

Table S3. B3LYP/6-311+G(3df,2pd) optimized geometry of 1,2-butadiene

Figure S1. Matrix-isolation FTIR spectrum showing formation of $\mathrm{HBr}$ in the 800-1000 K pyrolysis of 1-bromo-2-butyne

Figure S2. Matrix-isolation FTIR spectrum showing formation of vinylacetylene and propyne in the $800-1000 \mathrm{~K}$ pyrolysis of 1-bromo-2-butyne

Figure S3. Matrix-isolation FTIR spectrum showing formation of vinylacetylene and butatriene in the 800-1000 K pyrolysis of 1-bromo-2-butyne 
Table S1. B3LYP/6-311+G(3df,2pd) optimized geometry of the lowest energy ${ }^{2} \mathrm{~A}^{\prime}$ conformer of the 2-butyn-1-yl radical

$\begin{array}{lccc}\text { Atom } & \mathrm{X} & \mathrm{Y} & \mathrm{Z} \\ \mathrm{C} & -1.9648840 & -0.0000020 & 0.0014640 \\ \mathrm{H} & -2.3519900 & -0.0028280 & 1.0240700 \\ \mathrm{H} & -2.3639890 & -0.8819530 & -0.5030080 \\ \mathrm{H} & -2.3640220 & 0.8846520 & -0.4982030 \\ \mathrm{C} & -0.5137620 & 0.0000330 & -0.0068930 \\ \mathrm{C} & 0.7082950 & 0.0000080 & -0.0016760 \\ \mathrm{C} & 2.0734640 & -0.0000100 & 0.0021400 \\ \mathrm{H} & 2.6306470 & -0.9263060 & 0.0034660 \\ \mathrm{H} & 2.6306730 & 0.9262700 & 0.0034710\end{array}$

Table S2. B3LYP/6-311+G(3df,2pd) optimized geometry of 2-butyne

$\begin{array}{lccc}\text { Atom } & \mathrm{X} & \mathrm{Y} & \mathrm{Z} \\ \mathrm{C} & 0.0000000 & 0.0000000 & 2.0570750 \\ \mathrm{H} & 0.0002610 & 1.0175660 & 2.4513470 \\ \mathrm{H} & 0.8811070 & -0.5090090 & 2.4513470 \\ \mathrm{H} & -0.8813680 & -0.5085570 & 2.4513470 \\ \mathrm{C} & 0.0000000 & 0.0000000 & 0.6004830 \\ \mathrm{C} & 0.0000000 & 0.0000000 & -0.6004830 \\ \mathrm{C} & 0.0000000 & 0.0000000 & -2.0570750 \\ \mathrm{H} & 0.8813680 & -0.5085570 & -2.4513470 \\ \mathrm{H} & -0.8811070 & -0.5090090 & -2.4513470 \\ \mathrm{H} & -0.0002610 & 1.0175660 & -2.4513470\end{array}$


Table S3. B3LYP/6-311+G(3df,2pd) optimized geometry of 1,2-butadiene

$\begin{array}{lccc}\text { Atom } & \mathrm{X} & \mathrm{Y} & \mathrm{Z} \\ \mathrm{C} & 1.9341100 & -0.2135820 & -0.0000960 \\ \mathrm{H} & 2.4749610 & -0.3803570 & 0.9239220 \\ \mathrm{H} & 2.4746900 & -0.3805760 & -0.9242290 \\ \mathrm{C} & 0.6913390 & 0.1733700 & 0.0003050 \\ \mathrm{C} & -0.5523310 & 0.5574830 & -0.0001580 \\ \mathrm{H} & -0.7565900 & 1.6247610 & -0.0002000 \\ \mathrm{C} & -1.7433750 & -0.3638890 & 0.0000190 \\ \mathrm{H} & -2.3674450 & -0.1884570 & 0.8788100 \\ \mathrm{H} & -2.3679630 & -0.1880890 & -0.8783300 \\ \mathrm{H} & -1.4361110 & -1.4075710 & -0.0003910\end{array}$




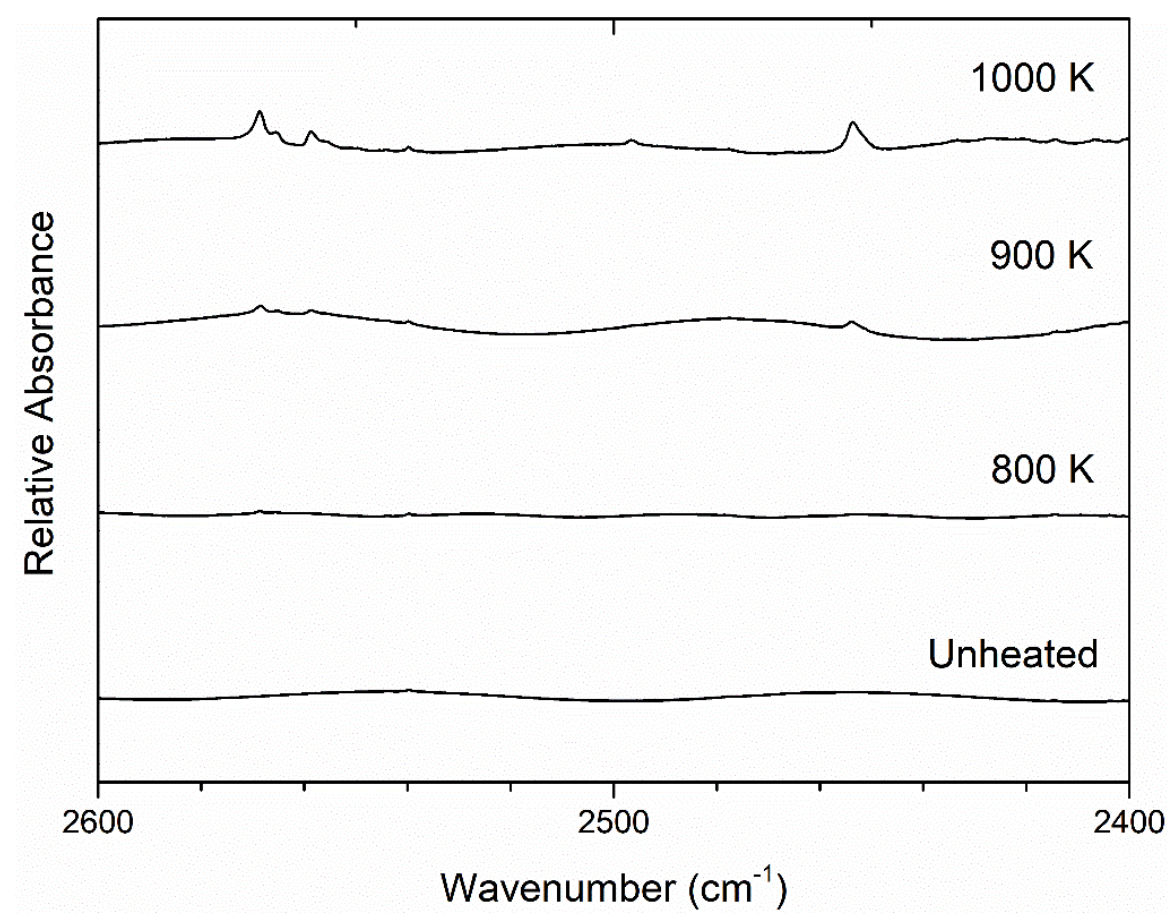

Figure S1. Matrix-isolation FTIR spectra collected following pyrolysis of 1-bromo-2butyne at various temperatures. Bands at 2569, 2558, and $2496 \mathrm{~cm}^{-1}$ show $\mathrm{HBr}$ monomer and dimer.

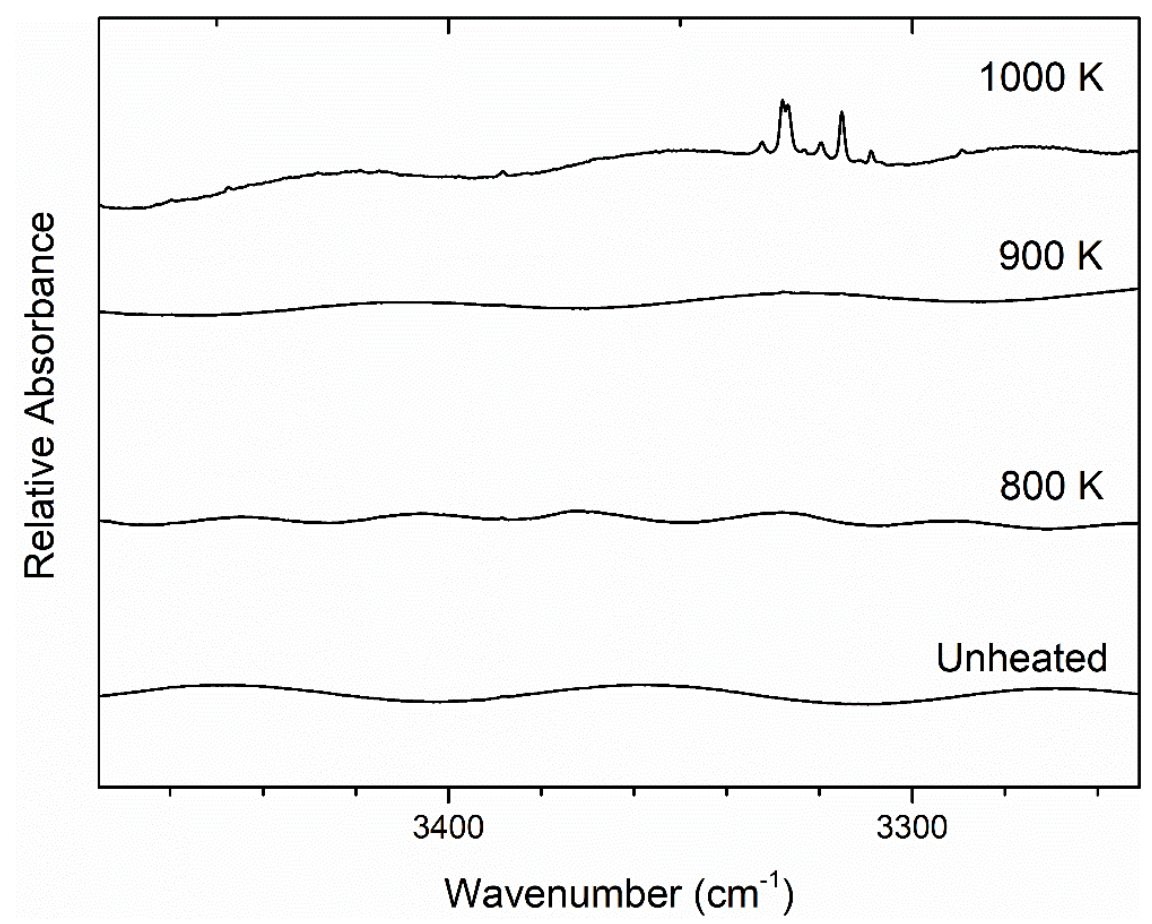

Figure S2. Matrix-isolation FTIR spectra collected following pyrolysis of 1-bromo-2butyne at various temperatures. Bands in the vicinity of $3320 \mathrm{~cm}^{-1}$ show vinylacetylene and propyne. 


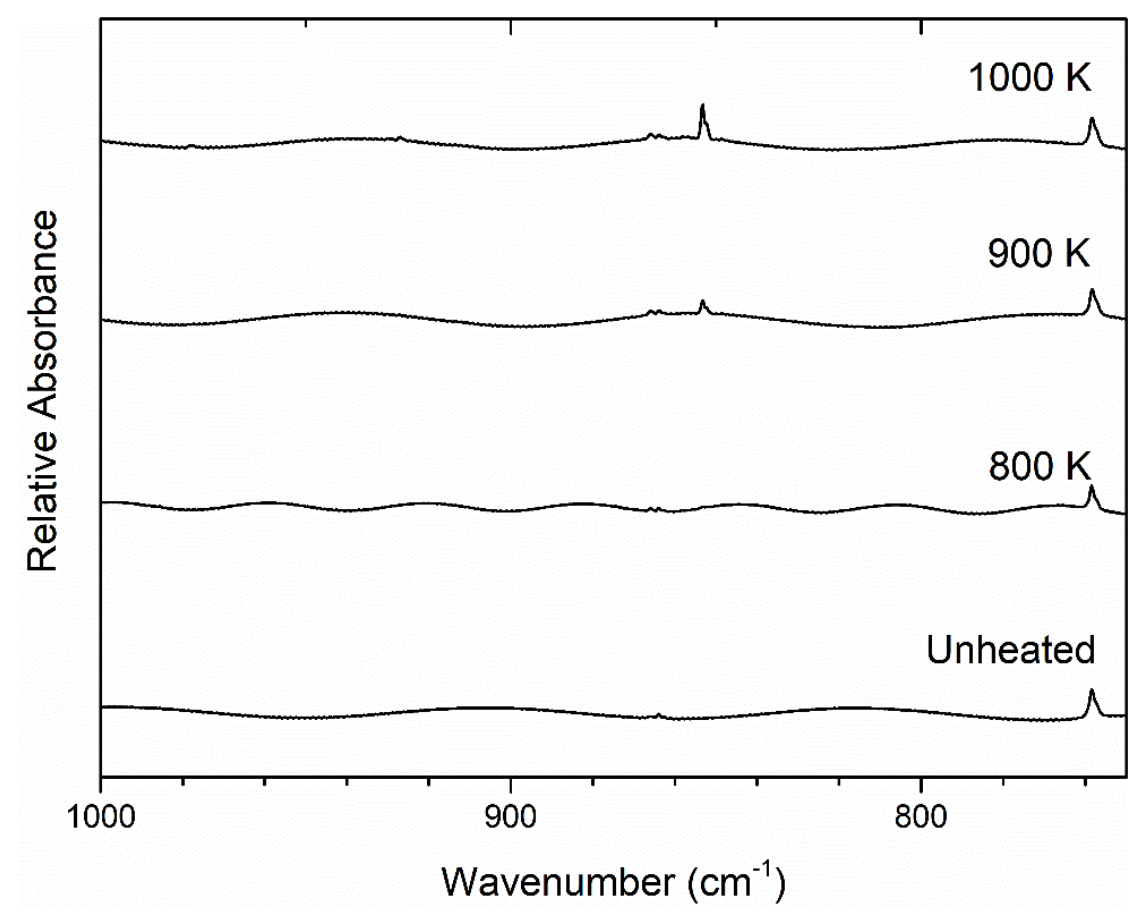

Figure S3. Matrix-isolation FTIR spectra collected following pyrolysis of 1-bromo-2butyne at various temperatures. Bands corresponding to butatriene $\left(853 \mathrm{~cm}^{-1}\right)$ vinylacetylene (978 and $927 \mathrm{~cm}^{-1}$ ) become more prominent as pyrolysis temperature increases.

Full citation for reference 30 .

Frisch, M. J.; Trucks, G. W.; Schlegel, H. B.; Scuseria, G. E.; Robb, M. A.; Cheeseman, J. R.; Scalmani, G.; Barone, V.; Mennucci, B.; Petersson, G. A.; Nakatsuji, H.; Caricato, M.; Li, X.; Hratchian, H. P.; Izmaylov, A. F.; Bloino, J.; Zheng, G.; Sonnenberg, J. L.; Hada, M.; Ehara, M.; Toyota, K.; Fukuda, R.; Hasegawa, J.; Ishida, M.; Nakajima, T.; Honda, Y.; Kitao, O.; Nakai, H.; Vreven, T.; J.A. Montgomery, J.; Peralta, J. E.; Ogliaro, F.; Bearpark, M.; Heyd, J. J.; Brothers, E.; Kudin, K. N.; Staroverov, V. N.; Kobayashi, R.; Normand, J.; Raghavachari, K.; Rendell, A.; Burant, J. C.; Iyengar, S. S.; Tomasi, J.; Cossi, M.; Rega, N.; Millam, J. M.; Klene, M.; Knox, J. E.; Cross, J. B.; Bakken, V.; Adamo, C.; Jaramillo, J.; Gomperts, R.; Stratmann, R. E.; Yazyev, O.; Austin, A. J.; Cammi, R.; Pomelli, C.; Ochterski, J. W.; Martin, R. L.; Morokuma, K.; Zakrzewski, V. G.; Voth, G. A.; Salvador, P.; Dannenberg, J. J.; Dapprich, S.; Daniels, A. D.; Farkas, Ö.; Foresman, J. B.; Ortiz, J. V.; Cioslowski, J.; Fox, D. J. Gaussian 09, Revision B.01, Gaussian, Inc.: Wallingford, CT, 2010. 